\title{
Inatividade física, comportamento sedentário e excesso de peso corporal associados à condição socioeconômica em jovens
}

CDD. 20.ed. 616.398

796.05

\author{
Aldemir SMITH-MENEZES ${ }^{* * *}$ \\ Maria de Fátima da Silva DUARTE* \\ Roberto J erônimo dos Santos SILVA***
}

${ }^{*}$ Centro de Desportos, Universidade Federal de Santa Catarina. ${ }^{* *}$ Instituto Federal de Educação, Ciência e Tecnologia de Sergipe. ***Universidade Federal de Sergipe.

\section{Resumo}

Este trabalho teve por objetivo verificar a associação entre fatores da condição socioeconômica com a inatividade fisica, comportamento sedentário e excesso de peso corporal em jovens. A amostra deste estudo foi composta por 758 sujeitos, retirada da população de jovens em condição de alistamento militar. As variáveis independentes do estudo foram: classe econômica, estado civil, escolaridade, ser trabalhador. A inatividade física, exposição ao comportamento sedentário e excesso de peso corporal foram utilizados como variáveis dependentes. Após ajuste, verificou-se associação entre a inatividade física e os indicadores de condição econômica escolaridade $(\mathrm{RP}=1,42 ; \mathrm{IC} 95 \%=1,00-2,01)$ e ser trabalhador $(\mathrm{RP}=1,67 ; \mathrm{IC}=95 \% 1,21-2,32) .0$ excesso de peso corporal associou-se com as classes econômicas "C" (RP =2,60; IC 95\% =1,40-4,84) e "D/E" (RP =2,08; IC 95\% = 1,26$3,44)$. Conclui-se que jovens com maior escolaridade e que não trabalhavam foram mais inativos fisicamente e que os que estavam em menor condição econômica mostraram-se com excesso de peso corporal.

UNITERMOS: Atividade motora; Sobrepeso; "Status" socioeconômico.

\section{Introdução}

A evolução tecnológica ocorrida desde a metade do século passado e as alteraçóes no campo social, político e econômico, bem como, a urbanização das cidades proporcionaram mudanças nas condiçóes de vida e de saúde das pessoas (DuCHIADE, 1999) e, como consequência, na exposição ao comportamento sedentário (CS), no nível insuficiente de atividade física (NIAF) e no excesso de peso corporal em nível populacional (KATZMArZYK \& MAsOn, 2009; Malina \& Little, 2008).

Havendo fortes evidências da relação entre prática regular de $\mathrm{AF}$ e os aspectos do processo saúde-doença (Löllgen, BÖCKEnHoff \& KNAPp, 2009; U.S. Department of Health and Human SERVICES, 2008), parece que a condição socioeconômica representa um fator importante em favorecer um estilo de vida fisicamente ativo em adolescentes (Stalsberg \& Pedersen, 2010).

Nesta perspectiva, investigações realizadas no Brasil, com vários subgrupos populacionais, apontam para a associação entre atividade física e a menor renda familiar, menor escolaridade, sexo masculino e com a renda "per capita" (MARCONDELli, Costa \& Schmitz, 2008; Oehlschlaeger, Pinheiro, Horta, Gelatti \& San'tana, 2004; Sávio, Costa, Schmitz \& Silva, 2008). Por outro lado, a exposição ao CS mostrou-se associado aos jovens com maior idade, não trabalhadores e de maior escolaridade (CuNHA, Peixoto, Jardim \& Alexandre, 2008; Farias JúNIOR, 2006, 2008).

Fato interessante a ser ressaltado é que quando considerado o excesso de peso corporal há uma tendência a ser verificada associação direta com a condição socioeconômica em jovens masculinos (ARAúJO, KONRAD, Rabacow, Graup, Amboni \& Farias Júnior, 2007), o que pode influenciar diretamente não só na adoção de comportamentos de risco para a obesidade, mas também tende a contribuir para uma vida adulta caracterizada pela obesidade.

Sendo assim, esse estudo se justifica pela possibilidade de inserção no mercado de trabalho e alistamento militar para a maioria dos sujeitos, bem como, em decorrência de mudanças comportamentais que ocorrem no final da adolescência. 
Neste sentido, este trabalho tem por objetivo verificar a associação de fatores da condição socioeconômica com a inatividade física, comportamento sedentário

\section{Método}

\section{População e amostra}

Foi realizada uma pesquisa caracterizada como epidemiológica de corte transversal com rapazes em idade de alistamento militar. Os dados foram coletados no ano de 2007 no $28^{\circ}$ Batalhão de Caçadores (28 BC) - Exército Brasileiro, localizado na cidade de Aracaju, Sergipe, Brasil, sob autorização do Comando Maior da instituição. De acordo com informações dessa entidade, a população alistada em 2007 para concorrer ao serviço militar obrigatório foi de 5.114 sujeitos.

A seleção da amostra foi realizada mediante processo aleatório simples em dois estágios. No primeiro, foi realizado o sorteio de três dias da semana (terça, quinta e sexta-feira) para coletar os dados; no segundo, sortearam-se 30 sujeitos para responder ao instrumento em cada dia.

Para a estimativa do tamanho amostral, considerou-se um Intervalo de Confiança de 95\%, uma prevalência estimada para adolescentes em 50\% e erro tolerável da amostragem em quatro pontos percentuais. A partir destes elementos a amostra foi estimada em 645 sujeitos, prevendo $10 \%$ de eventuais perdas.

Os critérios adotados para inclusão dos participantes na pesquisa foram os seguintes: estar disposto a participar da pesquisa, assinar um termo de consentimento livre e esclarecido e não ser classificado como "refratário" (sujeito com idade superior a 18 anos).

Um questionário foi aplicado para 809 sujeitos, sendo excluídos 31 por serem classificados como "refratários" e 20 por não preenchimento de questôes importantes totalizando a amostra final de 758 (perdas de 6,3\%).

\section{Instrumentos e coleta de dados}

Foram coletadas informações referentes a aspectos socioeconômicos (escolaridade, estado civil, trabalho, classe econômica), Índice de Massa Corporal (massa corporal e estatura) e comportamental (nível de atividade física e exposição ao comportamento e excesso de peso corporal em jovens sergipanos no período do alistamento militar obrigatório no Estado de Sergipe.

sedentário), mediante aplicação de questionário auto-administrado.

A escolaridade foi mensurada em anos completos de estudo, de forma que as respostas foram categorizada $<9$ anos de estudo (ensino fundamental incompleto) e $\geq 9$ anos de estudo (ensino fundamental completo). O estado civil também foi classificado em duas categorias: "vivendo com parceiro" e "vivendo sem parceiro". O trabalho foi dicotomizado em "sim" (trabalhador) e "não" (não trabalhador).

Para a identificação da classe econômica foram empregadas questôes relativas ao Critério de Classificação Econômica Brasil (ABEP, 2000). Esse instrumento de coleta visa classificar a população brasileira de acordo com seu o poder de compras dentro de uma escala de "A" a "E". Para melhor organização dos dados, para este estudo distribuiuse em três categorias: Classe "A/B" (elevada), Classe "C" (intermediária) e Classe "D/E" (baixa).

$\mathrm{Na}$ identificação do IMC foram mensuradas a massa corporal e a estatura. $\mathrm{Na}$ análise descritiva o IMC foi classificado em três categorias: baixo peso $\left(<18,5 \mathrm{~kg} / \mathrm{m}^{2}\right)$, eutrófico $\left(18,5-24,9 \mathrm{~kg} / \mathrm{m}^{2}\right)$ e excesso de peso corporal $\left(\geq 25,0 \mathrm{~kg} / \mathrm{m}^{2}\right)$. Para a análise dicotômica foi utilizada a classificação peso normal $\left(<25,0 \mathrm{~kg} / \mathrm{m}^{2}\right)$ e excesso de peso corporal $\left(\geq 25,0 \mathrm{~kg} / \mathrm{m}^{2}\right)$.

Para avaliar o nível de atividades físicas (NAF) em diferentes dimensóes (caminhadas e esforços físicos de intensidades moderada e vigorosa), foi utilizada a versão curta do Questionário Internacional de Atividades Físicas (IPAQ) (Matsudo, Araújo, Matsudo, Andrade, Andrade, Oliveira \&Bragion, 2001). Para a classificação do NAF foi considerada a recomendação internacional de " $<150 \mathrm{~min} / \mathrm{sem}$ " (nível insuficiente de atividades físicas - NIAF) e " $\geq 150$ min/sem” (ativo) (Haskell, Lee, Pate, Powell, Blair, Franklin, Macera, Heath, Thompson \& Bauman, 2007). Para a exposição ao CS (tempo gasto assistindo TV, fazendo uso do computador ou jogando videogame) foi utilizado como critério " $<2$ horas/ dia" e " $\geq 2$ horas/dia" (Tremblay, Leblanc, Janssen, Kho, Hicks, Murumets, Colley \& Duggan, 2011). 


\section{Tratamento estatístico}

$\mathrm{Na}$ análise dos dados foi realizada a estatística descritiva (frequência absoluta e relativa). O teste do qui-quadrado foi usado para avaliar heterogeneidade dos dados ou a tendência linear. Para avaliar a associação bruta entre indicadores selecionados de condiçóes socioeconômicas (escolaridade, trabalho, classe econômica, estado civil) com as variáveis dependentes NIAF, exposição ao CS e excesso de peso corporal utilizou-se à medida da razão de prevalência (RP) com intervalos de confiança de 95\% (IC 95\%), por meio da regressão de Poisson. O teste de Wald para heterogeneidade foi utilizado para verificar o nível de significância da relação entre as variáveis dependentes e independentes.

\section{Resultados}

Todos os participantes eleitos para o estudo nasceram no ano de 1989 (informaram ter 18 anos completos ou incompletos). A prevalência de jovens com nível insuficiente de atividade física foi de $37,3 \%$, expostos ao comportamento sedentário $(\geq 2$ horas/dia assistindo TV, no computador ou jogando videogame) foi de 79,8\%, com ensino fundamental completo $81,9 \%$ e pertencente à classe econômica D/E foi $61,2 \%$.

A TABELA 1 descreve a análise de heterogeneidade ou tendência linear entre as variáveis dependentes (exposição ao CS, NIAF e IMC) e os indicadores selecionados de condição socioeconômica. A exposição ao CS foi mais evidente entre os sujeitos com maior escolaridade, com menor classe econômica ( $\mathrm{p}$ $=0,013$ ), vivendo sem parceiros e não trabalhador. Cerca de $40 \%$ dos sujeitos com maior escolaridade e $41 \%$ dos jovens que informaram não trabalhar mostraram-se com NIAF. Aproximadamente 24\%
$\mathrm{Na}$ análise multivariável buscou-se ajustar às variáveis dependentes atividade física (ativo $=0 \mathrm{e}$ NIAF = 1), exposição ao CS $(<2 \mathrm{~h} / \mathrm{dia}=0 \mathrm{e} \geq 2 \mathrm{~h} /$ $\mathrm{dia}=1$ ) e índice de massa corporal (peso normal $=0 \mathrm{e}$ excesso de peso corporal $=1$ ) aos quatro indicadores selecionados de condição socioeconômica do estudo (escolaridade, trabalho, classe econômica, e estado civil). Para o gerenciamento dos dados foi utilizado o pacote estatístico SPSS versão 15.0. O nível de significância adotado foi $\leq 5 \%$.

Esta pesquisa seguiu os princípios éticos presentes na Declaração de Helsinque. Os protocolos de pesquisa foram avaliados e aprovados pelo Comitê de Ética em Pesquisa com Seres Humanos da Universidade Tiradentes (Processo ${ }^{\circ}{ }^{\text {. }}$ 1612/07).

dos sujeitos com excesso de peso corporal pertenciam a classe econômica "C" ou viviam com um parceiro. Por outro lado, a maioria dos jovens em qualquer indicador da condição socioeconômica pertencia ao grupo dos eutróficos.

A TABELA 2 apresenta a associação bruta entre as variáveis dependentes (nível insuficiente de atividade física, CS e excesso de peso corporal) e as variáveis independentes (escolaridade, classe econômica, estado civil e trabalhado). O nível insuficiente de atividade física foi mais frequente entre os jovens com maior escolaridade (RP 1,42; IC 95\% 1,01-2,00) e entre os que não trabalham (RP 1,65; IC 95\% 1,202,27). A exposição ao CS não apresentou associação com os indicadores da condição socioeconômica na análise bruta. $\mathrm{O}$ excesso de peso corporal foi mais evidente entre os sujeitos das classes econômicas "C" (RP 2,50; IC 95\% 1,36-4,62) e "D/E” (RP 2,01; IC $95 \%$ 1,22-3,29). 
SMITH-MENEZES, A.; DUARTE, M.F.S. \& SILVA, R.J .S.

TABELA 1 - Características dos jovens por nível deatividadefísica (NAF), exposição ao comportamento sedentário (CS) e Índice de Massa Corporal (IMC), segundo condição socioeconômica.

${ }^{*} p \leq 0,005$.

\begin{tabular}{|c|c|c|c|c|c|c|c|c|}
\hline & \multirow[b]{2}{*}{$\mathbf{N}$} & \multicolumn{2}{|c|}{ Exposição ao CS (\%) } & \multicolumn{2}{|c|}{ NAF (\%) } & \multicolumn{3}{|c|}{ IMC (\%) } \\
\hline & & $<2 \mathrm{~h} / \mathrm{dia}$ & $\geq 2 \mathrm{~h} / \mathrm{dia}$ & $\begin{array}{c}\geq 150 \\
\mathrm{~min} / \mathrm{sem}\end{array}$ & $\begin{array}{c}<150 \\
\mathrm{~min} / \mathrm{sem}\end{array}$ & $\begin{array}{c}\text { Baixo } \\
\text { Peso }\end{array}$ & Eutrófico & $\begin{array}{c}\text { Excesso } \\
\text { de peso } \\
\text { Corporal }\end{array}$ \\
\hline \multicolumn{9}{|l|}{ Escolaridade } \\
\hline$<8$ anos & 75 & 24,0 & 76,0 & 72,26 & 27,74 & 16,79 & 67,15 & 15,33 \\
\hline$\geq 8$ anos & 683 & 17,4 & 82,6 & 60,55 & 39,45 & 12,24 & 70,85 & 16,91 \\
\hline p-valor & & \multicolumn{2}{|c|}{0,160} & \multicolumn{2}{|c|}{$0,033^{*}$} & \multicolumn{3}{|c|}{0,437} \\
\hline \multicolumn{9}{|l|}{ Classe Econômica } \\
\hline Classe A/B & 201 & 14,4 & 85,6 & 62,19 & 37,81 & 15,92 & 74,63 & 9,45 \\
\hline Classe C & 93 & 9,7 & 90,3 & 61,29 & 38,71 & 6,45 & 69,89 & 23,66 \\
\hline Classe D/E & 464 & 8,0 & 92,0 & 63,15 & 36,85 & 13,15 & 68,32 & 18,32 \\
\hline p-valor & & \multicolumn{2}{|c|}{$0,038^{*}$} & \multicolumn{2}{|c|}{0,932} & \multicolumn{3}{|c|}{$0,005^{*}$} \\
\hline \multicolumn{9}{|l|}{ Estado Civil } \\
\hline Vivendo com parceiro & 21 & 28,6 & 71,4 & 47,62 & 52,38 & 33,33 & 42,86 & 23,81 \\
\hline Vivendo sem parceiro & 737 & 9,4 & 90,6 & 63,09 & 36,91 & 12,48 & 70,96 & 16,42 \\
\hline p-valor & & \multicolumn{2}{|c|}{$0,004^{*}$} & \multicolumn{2}{|c|}{0,148} & \multicolumn{3}{|c|}{$0,008^{*}$} \\
\hline \multicolumn{9}{|l|}{ Trabalhador } \\
\hline $\operatorname{Sim}$ & 180 & 12,8 & 87,2 & 75,00 & 25,00 & 17,22 & 65,00 & 17,22 \\
\hline Não & 578 & 9,0 & 91,0 & 58,82 & 41,18 & 11,76 & 71,80 & 16,44 \\
\hline p-valor & & \multicolumn{2}{|c|}{0,138} & \multicolumn{2}{|c|}{$<0,001^{*}$} & \multicolumn{3}{|c|}{0,372} \\
\hline
\end{tabular}

TABELA 2 - Análise da razão de prevalência (RP) bruta e intervalos de 95\% confiança (IC 95\%) para o nível insuficiente de atividade física (NIAF), exposição ao comportamento sedentário (CS) e excesso de peso corporal, por condição socioeconômica, Sergipe, Brasil.

${ }^{*} p<0,05$.

\begin{tabular}{|c|c|c|c|c|c|c|}
\hline \multirow[t]{2}{*}{ Variáveis } & \multicolumn{2}{|c|}{$\begin{array}{c}\text { NIAF } \\
(<150 \mathrm{~min} / \mathrm{sem})\end{array}$} & \multicolumn{2}{|c|}{$\begin{array}{l}\text { Exposição ao CS } \\
\quad(>2 \mathrm{~h} / \mathrm{dia})\end{array}$} & \multicolumn{2}{|c|}{$\begin{array}{l}\text { Excesso de Peso Corporal } \\
\left(\mathrm{IMC}>25 \mathrm{k} / \mathrm{m}^{2}\right)\end{array}$} \\
\hline & RP (IC 95\%) & p-valor & RP (IC 95\%) & p-valor & RP (IC 95\%) & p-valor \\
\hline \multicolumn{7}{|l|}{ Escolaridade } \\
\hline$<8$ anos & 1 & \multirow{2}{*}{$0,043^{*}$} & 1 & \multirow{2}{*}{0,659} & 1 & \multirow{2}{*}{0,764} \\
\hline$\geq 8$ anos & $1,42(1,01-2,00)$ & & $1,05(0,86-1,27)$ & & $1,07(0,68-1,70)$ & \\
\hline
\end{tabular}

Classe Econômica

\begin{tabular}{|c|c|c|c|c|c|c|}
\hline Classe A/B & 1 & & 1 & & 1 & \\
\hline Classe C & $1,02(0,69-1,52)$ & 0,957 & $1,06(0,90-1,28)$ & 0,723 & $2,50(1,36-4,62)$ & $0,008^{*}$ \\
\hline Classe D/E & $0,98(0,74-1,28)$ & & $1,08(0,81-1,37)$ & & $2,01(1,22-3,29)$ & \\
\hline
\end{tabular}

\section{Estado Civil}

$\begin{array}{ccccccc}\text { Vivendo com parceiro } & 1 & 1 & 1 & 0,447 \\ \text { Vivendo sem parceiro } & 0,71(0,39-1,29) & 0,255 & 1,27(0,76-2,12) & 0,362 & 0,71(0,29-1,73) & \end{array}$

\section{Trabalhador}

\begin{tabular}{|c|c|c|c|c|c|c|}
\hline Sim & 1 & & 1 & & 1 & \\
\hline Não & $1,65(1,20-2,27)$ & 0,002 & $1,04(0,87-1,25)$ & 0,041 & $0,91(0,61-1,35)$ & \\
\hline
\end{tabular}

414 • Rev. bras. Educ. Fís. Esporte, São Paulo, v.26, n.3, p.411-18, jul./ set. 2012 
A TABELA 3 apresenta a análise ajustada entre o NIAF, exposição ao CS e excesso de peso corporal e as variáveis independentes do modelo. Após o ajuste os indicadores de condição econômica, escolaridade (RP 1,42; IC 95\% 1,00-2,01) e ser trabalhador (RP 1,67; IC 95\% 1,21-2,32) continuaram associados ao desfecho NIAF. A exposição ao CS não se associou com qualquer variável. $\mathrm{O}$ excesso de peso permaneceu sendo mais provável entre os sujeitos das classes econômicas "C" (RP 2,60; IC 95\% 1,40-4,84) e “D/E” (RP 2,08; IC 95\% 1,26-3,44).

TABELA 3 - Análise da razão de prevalência (RP) ajustada e intervalos de 95\% confiança (IC 95\%) do nível insuficiente de atividade física (NIAF), exposição ao comportamento sedentário (CS) e excesso de peso corporal, por condição socioeconômica, Sergipe, Brasil.

\begin{tabular}{|c|c|c|c|c|c|c|}
\hline \multirow[t]{2}{*}{ Variáveis } & \multicolumn{2}{|c|}{$\begin{array}{l}\text { Inatividade Física } \\
(<150 \mathrm{~min} / \mathrm{sem})\end{array}$} & \multicolumn{2}{|c|}{$\mathrm{CS}(>2 \mathrm{~h} / \mathrm{dia})$} & \multicolumn{2}{|c|}{$\begin{array}{l}\text { Excesso de Peso Corporal } \\
\left(\text { IMC }>25 \mathrm{k} / \mathrm{m}^{2}\right)\end{array}$} \\
\hline & RP (IC 95\%) & p-valor & RP (IC 95\%) & p-valor & RP (IC 95\%) & p-valor \\
\hline \multicolumn{7}{|l|}{ Escolaridade } \\
\hline$<8$ anos & 1 & \multirow{2}{*}{$0,050^{*}$} & 1 & \multirow{2}{*}{0,828} & 1 & \multirow{2}{*}{0,962} \\
\hline$\geq 8$ anos & $1,42(1,00-2,01)$ & & $1,02(0,84-1,25)$ & & $0,99(0,62-1,58)$ & \\
\hline \multicolumn{7}{|l|}{ Classe Econômica } \\
\hline Classe A/B & 1 & \multirow{3}{*}{0,837} & 1 & \multirow{3}{*}{0,806} & 1 & \multirow{3}{*}{$0,006^{*}$} \\
\hline Classe C & $0,95(0,64-1,42)$ & & $1,04(0,80-1,36)$ & & $2,60(1,40-4,84)$ & \\
\hline Classe D/E & $0,92(0,70-1,21)$ & & $1,06(0,89-1,27)$ & & $2,08(1,26-3,44)$ & \\
\hline \multicolumn{7}{|l|}{ Estado Civil } \\
\hline Vivendo com parceiro & 1 & \multirow{2}{*}{0,063} & 1 & \multirow{2}{*}{0,431} & 1 & \multirow{2}{*}{0,309} \\
\hline Vivendo sem parceiro & $0,56(0,30-2,32)$ & & $1,23(0,73-2,07)$ & & $0,62(0,25-1,55)$ & \\
\hline \multicolumn{7}{|l|}{ Trabalhador } \\
\hline Sim & 1 & \multirow{2}{*}{$0,002^{*}$} & 1 & \multirow{2}{*}{0,783} & 1 & \multirow{2}{*}{0,506} \\
\hline Não & $1,67(1,21-2,32)$ & & $1,03(0,86-1,23)$ & & $0,87(0,58-1,31)$ & \\
\hline
\end{tabular}

\section{Discussão}

O presente estudo descreve a associação entre a NIAF, exposição ao CS e excesso de peso corporal com a condição socioeconômica em jovens masculinos. Foi conduzido com uma amostra representativa, predominantemente, com mais de oito anos de estudo, vivendo sem parceiro, não trabalhadora e de baixa classe econômica, diferenciando-se de outras investigaçōes por ser conduzido com uma população específica de jovens da mesma idade e sexo.

São poucas as pesquisas realizadas no Brasil buscando identificar os fatores associados ao NIAF, exposição ao CS (Dumith, 2009) e excesso de peso corporal (ARAújo et al., 2007) em adolescentes. Da mesma forma, não foram encontrados estudos que analisassem um subgrupo populacional de jovens com 18 anos de idade na Região Nordeste do Brasil.

Revisões sistemáticas apontam que a prática habitual de atividades físicas e o CS apresentam relação direta com algumas variáveis sociodemográficas (STALSBERG \& PEDERSEN, 2010), comportamentais (FARIAS Júnior, Mendes \& Barbosa, 2007; Farias Júnior, Nahas, Barros, Loch, Oliveira, De Bem \& Lopes, 2009) e outras variáveis ambientais (FERREIRA, HorST, Wendel-Vos, Kremers, VAn Lenthe \& Brug, 2006; Sallis, Prochaska \& Taylor, 2000; Van Der Horst, Paw, Twisk \& VAN Mechelen, 2007).

Apesar de ser difícil a comparação em virtude do instrumento utilizado, no modelo ajustado deste estudo foi verificada associação do NIAF dos adolescentes com o fato de não ser trabalhador. Outros manuscritos encontraram resultados semelhantes (FARIAS JúnIOR, 2008; TENÓRIO, Barros, Tassitano, Bezerra, Tenório \& Hallal, 2010) ou não encontraram relação com esta variável (FArias Júnior et al., 2009). Tais resultados podem estar relacionados à elevada prevalência na 
exposição ao CS sugerindo intervenções em locais onde convivem grupos de adolescentes, apesar de evidências sugerirem independência nas variáveis.

A relação do NIAF com a maior escolaridade encontrada mostrou-se contrária a de outros estudos conduzidos no Brasil (OeHLSChlaEger et al., 2004; TenÓRIO et al., 2010). Por outro lado, estudos mostraram que jovens mais ativos (OliveIRA, SiLVA, SANTOS, Silva \& CONCEIÇÃO, 2010) tendem a apresentar menor escolaridade. Tais publicações parecem não apresentar um consenso quanto à relação do NIAF com a escolaridade e com o fato do adolescente ser trabalhador possivelmente em virtude do ponto de corte estabelecido ou devido ao instrumento utilizado para avaliar o nível de atividade física. Semelhante a outros estudos (Silva, NaHAs, Peres \& LOPES, 2009), a exposição ao CS não foi associada às variáveis consideradas, sugerindo novos estudos em que esta variável seja observada.

Foi verificado que o excesso de peso corporal apresentou relação com a classe econômica "C" e "D/E". Fato preocupante visto que pesquisas conduzidas com crianças (BAKer, Olsen \& Sorensen, 2007) e adolescentes (Bibbins-Domingo, Coxson, Pletcher, LigHTwOOD \& GOLDMAN, 2007) indicam que sobrepeso/obesidade adquirido nestes subgrupos populacionais pode ser projetado como causa de problemas cardiovasculares em idades mais avançadas. Da mesma forma, os resultados advertem que o elevado consumo calórico nas classes menos abastadas pode acarretar grande desequilíbrio nutricional, como visto em estudo realizado no Brasil (BATISTA Filho \& Rissin, 2003).

Evidências (Nunes, Figueiroa \& Alves, 2007) contrárias a esta investigação verificaram que a frequência de sobrepeso e obesidade é observada nos estratos sociais mais altos (A1, A2 e B) do que nas classes menores (C, D e E). Similarmente, estudo conduzido por Grujic, Cvejin, Nikolic, Dragnic, Jovanovic, Kvrgic e Travar (2009) com adultos jovens encontrou forte associação entre valores de sobrepeso/obesidade com o estado matrimonial, sexo masculino e menor nível educacional.

Num estudo que teve por objetivo avaliar a tendência de obesidade conforme o grau de escolaridade e prevalência de sobrepeso e obesidade em adultos e sua associação com o nível educacional, Marques-Vidal, Bovet, Paccaud e Chiolero (2007) verificaram que a prevalência de sobrepeso e obesidade foi inversamente associada com o nível educacional, sendo percebido um aumento anual de $0,45 \%, 0,29 \%$ e $0,06 \%$ para baixo, médio e alto níveis educacionais, respectivamente.

As principais limitações do estudo foram: 1) apesar do baixo custo e maior capacidade de operacionalização, a obtenção das informações por meio de questionário pode subestimar ou superestimar os resultados de pesquisas; 2) o delineamento transversal não permite avançar na análise quanto aos fatores que antecedem ou sucedem o desfecho; 3) diferença no instrumento de medida de AF e análise utilizada, dificultando a comparação com outras pesquisas.

Portanto, compreender que o contexto socioeconômico representa um dos mais importantes aspectos que podem favorecer estilos de vida cada vez mais ativos e redução no excesso de peso das pessoas, parece ser uma concepção mais interessante de ser abordada, sobretudo, quando se pensa em várias possibilidades de análise e interpretações mais coesas quanto às estratégias populacionais a serem desenvolvidas em termos de políticas públicas de saúde.

\section{Abstract}

Physical inactivity, sedentary behavior and overweight: association study with socioeconomic status in youth

The purpose of this study was to verify the association between socioeconomic factors and physical activity, sedentary behavior and overweight in youth. The sample was composed of 758 subjects from the population of initial military service. The independent variables were: socioeconomic class, marital status, educational level, and be worker. Physical Inactivity, exposures to sedentary behavior and be overweight were used as dependent variables. After adjustment, it was verified an association between physical inactivity and the indicators of economic status: education level $(P R=1.42,95 \% \mathrm{Cl}=1.00$ to 2.01$)$ and be worker $(\mathrm{PR}=1.67, \mathrm{Cl} 95 \%=1.21$ to 2.32). Overweight was associated with economic class " $\mathrm{C}$ " (PR $=2.60,95 \% \mathrm{Cl}=1.40$ to 4.84$)$ and "D/E" (PR $=2.08, \mathrm{Cl} 95 \%=1.26$ to 3.44$)$. It is concluded that youth with higher education level and not employed were more physically inactive. It was showed association between lower economic status and excess body weight.

UnIterms: Physical activity; Overweight; Socioeconomic status. 


\section{Resumen}

La inactividad física, el sedentarismo y el exceso de peso asociado con el nivel socioeconómico en los jóvenes

Este estudio tiene como objetivo verificar la asociación entre los factores socioeconómicos con inactividad física, el sedentarismo y el sobrepeso en los jóvenes. La muestra de este estudio consistió de 758 sujetos, la retirada de la población joven en situación de alistamiento militar. Las variables independientes del estudio fueron: clase económica, estado civil, educación, ser trabajador. La inactividad física, el sedentarismo y la exposición al exceso de peso se utilizaron como la variable dependiente. Después del ajuste, compruebe COU una asociación entre la inactividad física y los indicadores de la educación la situación económica ( $R P=1,42$, IC $95 \%=1,00$ a 2,01) y de ser un trabajador $(R P=1,67$, IC del 95\%: 1,21 a 2,32). El exceso de peso se asocia con las clases económicas "C" $(R P=2,60$, IC 95\% $=1,40$ a 4,84) y "D / E" $(R P=2,08, I C 95 \%=1,26$ a 3,44). Se llegó a la conclusión de que los jóvenes con más educación y que no estaban empleadas eran más inactivos físicamente y los que estaban mostraron una menor afección económica con exceso de peso corporal.

Palabras clave: Actividad física; Sobrepeso; Nivel socio-económico.

\section{Referências}

ASSOCIAÇÃO BRASILEIRA DE EMPRESAS DE PESQUISAS (ABEP). Critério de classificação sócio econômica: Brasil. São Paulo: 2000. Disponível em: <http://www.datavale-sp.com.br/CCEB.pdf>. Acesso em: 21 nov. 2007.

ARAÚJO V.C.; KONRAD L.M.; RABACOW, F.M.; GRAUP, S.; AMBONI, R.; FARIAS JÚNIOR, J.C. Prevalência de excesso de peso em adolescentes brasileiros: um estudo de revisão sistemática. Revista Brasileira de Atividade Física $\&$ Saúde, Pelotas, v.12, n.3, p.79-87, 2007.

BAKER J.L.; OLSEN, L.W.; SORENSEN, T.I.A. Childhood body-mass index and the risk of coronary heart disease in adulthood. The New England Journal of Medicine, Boston, v.357, p.2329-37, 2007.

BATISTA FILHO, M.; RISSIN, A. A transição nutricional no Brasil: tendências regionais e temporais. Cadernos de Saúde Pública, Rio de Janeiro, v.19, p.S181-S191, 2003. Suplemento 1.

BIBBINS-DOMINGO, K.; COXSON, P.; PLETCHER, M.J.; LIGHTWOOD, J.; GOLDMAN, L. Adolescent overweight and future adult coronary heart disease. The New England Journal of Medicine, Boston, v.357, p.2371-9, 2007.

CUNHA, I.C.; PEIXOTO, M.R.G.; JARDIM, P.C.B.V.; ALEXANDRE, V.P. Fatores associados à prática de atividade física na população adulta de Goiânia: monitoramento por meio de entrevistas telefônicas. Revista Brasileira de Epidemiologia, São Paulo, v.11, n.3, p.495-504, 2008.

DUCHIADE, M.P. População brasileira: um retrato em movimento. In: MINAYO, M.C.S (Org.). Os muitos Brasis: saúde e população na década de 80. 2.ed. São Paulo: Hucitec/Abrasco, 1999.

DUMITH, S.C. Physical activity in Brazil: a systematic review. Cadernos de Saúde Pública, Rio de Janeiro, v.25, p.S415S426, 2009. Suplemento 3.

FARIAS JUNIOR, J.C. Associação entre prevalência de inatividade física e indicadores de condição socioeconômica em adolescentes. Revista Brasileira de Medicina do Esporte, São Paulo, v.14, n.2, p.109-14, 2008.

Prevalência e fatores de influencia para inatividade física em adolescentes. Revista Brasileira de Ciências e Movimento, São Caetano do Sul, v.14, n.1, p.63-70, 2006.

FARIAS JÚNIOR, J.C.; MENDES, J.K.F.; BARBOSA, D.B.M. Associação entre comportamentos de risco à saúde em adolescentes. Revista Brasiliera de Cineantropometria e Desempenho Humano, Florianópolis, v.9, n.3, p.250-6, 2007. FARIAS JÚNIOR, J.C.; NAHAS, M.V.; BARROS, M.V.G.; LOCH, M.R.; OLIVEIRA, E.S.A.; DE BEM, M.F.L.; LOPES, A.S. Comportamentos de risco à saúde em adolescentes no Sul do Brasil: prevalência e fatores associados. Revista Panamericana de Salud Publica, Washington, v.25, n.4, p.344-52, 2009.

FERREIRA, I.; HORST, K.V.D.; WENDEL-VOS, W.; KREMERS, S.; VAN LENTHE, F.J.; BRUG, J. Environmental correlates of physical activity in youth: a review and update. Obesity Reviews, Osford, v.8, p.129-54, 2006. 
GRUJIC, V.; CVEJIN, M.M.; NIKOLIC, E.A.; DRAGNIC, N.; JOVANOVIC, V.M.; KVRGIC, S.; TRAVAR, S. Association between obesity and socioeconomic factors and lifestyle. Vojnosanitetski Pregled, Belgrade, v.66, n.9, p.705-10, 2009. HASKELL, W.L.; LEE, I-M.; PATE, R.R.; POWELL, K.E.; BLAIR, S.N.; FRANKLIN, B.A.; MACERA, C.A.; HEATH, G.W.; THOMPSON, P.D.; BAUMAN A. Physical activity and public health updated recommendation for adults from the American College of Sports Medicine and the American Heart Association. Circulation, Hagerstown, v.116, p.1081-93, 2007. KATZMARZYK, P.T.; MASON, C. The physical activity transition. Journal of Physical Activity and Health, Champaign, v.6, p.269-80, 2009.

LÖLLGEN, H.; BÖCKENHOFF, A.; KNAPP, G. Physical activity and all-cause mortality: an updated meta-analysis with different intensity categories. International Journal of Sports Medicine, Stuttgart, v.30, p.213-24, 2009.

MALINA, R.M.; LITTLE, B.B. Physical activity: the present in the context of the past. American Journal of Human Biology, New York, v.20, p.373-91, 2008.

MARCONDELLI, P.; COSTA, T.H.M.; SCHMITZ, B.A.S. Nível de atividade física e hábitos alimentares de universitários do $3^{\circ}$ ao $5^{\circ}$ semestres da área da saúde. Revista de Nutriçáo, Campinas, v.21, n.1, p.39-47, 2008.

MARQUES-VIDAL, P.; BOVET, P.; PACCAUD, F.; CHIOLERO, A. Changes of overweight and obesity in the adult Swiss population according to educational level, from 1992 to 2007. BMC Public Health, London, v.10, p.3-8, 2010. MATSUDO, S.; ARAÚJO, T.; MATSUDO, V.; ANDRADE, D.; ANDRADE, E.; OLIVEIRA, L.C.; BRAGION, G. Questionário Internacional de Atividade Física (IPAQ): estudo de validade e reprodutibilidade no Brasil. Revista Brasileira de Atividade Física \& Saúde, Pelotas, v.2, p.5-18, 2001.

NUNES, M.M.A.; FIGUEIROA, J.N.; ALVES, J.G.B. Excesso de peso, atividade física e hábitos alimentares entre adolescentes de diferentes classes econômicas em Campina Grande (PB). Revista da Associação Médica Brasileira, São Paulo, v.53, p.2, p.130-4, 2007. OEHLSCHLAEGER, M.H.K.; PINHEIRO, R.T.; HORTA, B.L.; GELATTI, C.; SAN’TANA, P. Prevalência e fatores associados ao sedentarismo em adolescentes de área urbana. Revista de Saúde Pública, São Paulo, v.38, n.2, p.157-63, 2004. OLIVEIRA, T.C.; SILVA, A.A.M.; SANTOS, C.J.N.; SILVA, J.S.; CONCEIÇÃO, S.I.O. Atividade física e sedentarismo em escolares da rede pública e privada de ensino em São Luís. Revista de Saúde Pública, São Paulo, v.44, n.6, p.996-1004, 2010. SALLIS, J.F.; PROCHASKA, J.J.; TAYLOR, W.C. A review of correlates of physical activity of children and adolescents. Medicine and Science in Sports and Exercise, Madison, v.32, n.5, p.963-75, 2000.

SÁVIO, K.E.O.; COSTA, T.H.M.; SCHMITZ, B.A.S.; SILVA, E.F. Sexo, renda e escolaridade associados ao nível de atividade física de trabalhadores. Revista de Saúde Pública, São Paulo, v.42, n.3, p.457-63, 2008.

SILVA, K.S.; NAHAS, M.V.; PERES, K.G.; LOPES, A.S. Factors associated with physical activity, sedentary behavior, and participation in physical education among high school students in Santa Catarina State, Brazil. Cadernos de Saúde Pública, Rio de Janeiro, v.25, p.2187-2200, 2009.

STALSBERG, R.; PEDERSEN, A.V. Effects of socioeconomic status on the physical activity in adolescents: a systematic review of the evidence. Scandinavian Journal of Medicine \& Science in Sports, Copenhagen, v.20, p.368-83, 2010. TENÓRIO, M.C.M.; BARROS, M.V.G.; TASSITANO, R.M.; BEZERRA, J.; TENÓRIO, J.M.; HALLAL, P.C. Atividade física e comportamento sedentário em adolescentes estudantes do ensino médio. Revista Brasileira de Epidemiologia, São Paulo, v.13, n.1, p.105-17, 2010.

TREMBLAY, M.S.; LEBLANC, A.G.; JANSSEN, I.; KHO, M.E.; HICKS, A.; MURUMETS, K.; COLLEY, R.C.; DUGGAN, M. Canadian sedentary behaviour guidelines for children and youth. Applied Physiology, Nutrition, and Metabolism, Ottawa, v.36, n.1, p.59-64, 2011.

U.S. DEPARTMENT OF HEALTH AND HUMAN SERVICES. Physical Activity Guidelines Advisory Committee. Physical activity guidelines advisory committee report, 2008. Washington: Physical Activity Guidelines Advisory Committee, 2008. VAN DER HORST, K.; PAW, M.C.; TWISK, J.W.; VAN MECHELEN, W. A brief review on correlates of physical activity and sedentariness in youth. Medicine and Science in Sports and Exercise, Madison, v.39, n.8, p.1241-50, 2007.

ENDEREÇO Aldemir Smith-Menezes Av. Eng. Gentil Tavares da Mota, 1166 50100-130 - Aracaju - SE - BRASIL e-mail: aldemirsmith@yahoo.com.br
Recebido para publicação: 30/ 01/ 2011

Revisado: 20/04/2012

Aceito: 18/05/2012 